\title{
Concurrent context-free grammars
}

\begin{abstract}
In this paper we study some properties of context-free concurrent grammars which are controlled by Petri nets under parallel firing strategies, i.e., the transitions of a Petri net fire simultaneously in different modes. These variants of control increase the computational power of generative devices. Moreover, they can be used as theoretical models for parallel computing devices.
\end{abstract}

Keyword: Petri nets; Parallel firing; Controlled grammars; Parallel computing 